\title{
Paired-termini Antisense RNA Mediated Inhibition of DoxR in Streptomyces peucetius ATCC 27952
}

\author{
Amit Kumar Chaudhary, Anaya Raj Pokhrel, Nguyen Thi Hue, Jin Cheol Yoo, and Jae Kyung Sohng
}

Received: 21 November 2014 / Revised: 25 March 2015 / Accepted: 1 April 2015

(C) The Korean Society for Biotechnology and Bioengineering and Springer 2015

In the 2015 issue of Biotechnology and Bioprocess Engineering (BBE), an error occurred in the research article: Amit Kumar Chaudhary, Anaya Raj Pokhrel, Nguyen Thi Hue, Jin Cheol Yoo, and Jae Kyung Sohng (2015) Pairedtermini Antisense RNA Mediated Inhibition of DoxR in Streptomyces peucetius ATCC 27952. Biotechnol. Bioprocess Eng. 20: 381-388.
Jae Kyung Sohng", Amit Kumar Chaudhary, Anaya Raj Pokhrel, Nguyen Thi Hue

Institute of Biomolecule Reconstruction, Department of BT-convergent Pharmaceutical Engineering, Sun Moon University, Asan 336-708, Korea Tel: +82-41-530-2246; Fax: +82-41-544-2919

E-mail: sohng@sunmoon.ac.kr

Jin Cheol Yoo

Department of Pharmacy, College of Pharmacy, Chosun University, Gwangju 501-759, Korea
- In the authors's affiliation

Original wording:

Jin Cheol Yoo

Institute of Biomolecule Reconstruction, Department of BT-convergent Pharmaceutical Engineering, Sun Moon University, Asan 336-708, Korea

Amit Kumar Chaudhary, Anaya Raj Pokhrel, Nguyen Thi Hue, Jae Kyung Sohng*

Department of Pharmacy, College of Pharmacy, Chosun University, Gwangju 501-759, Korea

Tel: +82-41-530-2246; Fax: +82-41-544-2919

E-mail: sohng@sunmoon.ac.kr

This should be replaced by:

Jae Kyung Sohng*, Amit Kumar Chaudhary, Anaya Raj Pokhrel, Nguyen Thi Hue

Institute of Biomolecule Reconstruction, Department of BT-convergent Pharmaceutical Engineering, Sun Moon University, Asan 336-708, Korea

Tel: +82-41-530-2246; Fax: +82-41-544-2919

E-mail: sohng@sunmoon.ac.kr

Jin Cheol Yoo

Department of Pharmacy, College of Pharmacy, Chosun University, Gwangju 501-759, Korea

Received: 15 July 2015 